\title{
Effects of Span Length and Additional Structure on Flow-Induced Transverse Vibration Characteristic of a Cantilevered Rectangular Prism
}

\author{
La Ode Ahmad Barata1 ${ }^{*}$, Takahiro Kiwata², Takaaki Kono², Toshiyuki Ueno ${ }^{3}$ \\ ${ }^{1}$ Graduate School of Natural Science and Technology, Kanazawa University, Kanazawa, Japan \\ ${ }^{2}$ School of Mechanical Engineering, Kanazawa University, Kanazawa, Japan \\ ${ }^{3}$ School of Electrical and Computer Engineering, Kanazawa University, Kanazawa, Japan \\ Email: *ahmadbarata@gmail.com
}

How to cite this paper: Barata, L.O.A., Kiwata, T., Kono, T. and Ueno, T. (2020) Effects of Span Length and Additional Structure on Flow-Induced Transverse Vibration Characteristic of a Cantilevered Rectangular Prism. Journal of Flow Control, Measurement \& Visualization, 8, 102-120.

https://doi.org/10.4236/jfcmv.2020.83006

Received: December 17, 2019

Accepted: March 3, 2020

Published: May 28, 2020

Copyright $\odot 2020$ by author(s) and Scientific Research Publishing Inc. This work is licensed under the Creative Commons Attribution International License (CC BY 4.0).

http://creativecommons.org/licenses/by/4.0/

\begin{abstract}
We consider the effects of the aspect ratio $L / H$ (where $L$ is the length of a prism, and $H$ is the height of a prism normal to the flow direction) and the size of additional structures (which are a plate and a fin on the surface of a prism) on a vibration characteristic of a cantilevered rectangular prism. The present research is intended to support the analysis of energy harvesting research on the flow-induced vibration in water flow using a magnetostrictive phenomenon. The prisms are constructed from stainless steel and mounted elastically to a plate spring attached to the ceiling wall of the water tunnel. The prisms with aspect ratios of $L / H \geq 5$ have reasonably identical vibration characteristics. However, the difference in the vibration characteristic appears distinctly on a rectangular prism with an aspect ratio of $L / H=2.5$. The rectangular prism with an aspect ratio of $L / H=10$ and a side ratio of $D / H=0.2$ has a stable and large response amplitude and oscillates with a lower velocity. The length of the added plate and the size of the added fin influence the velocity of vibration onset. If the length of the added plate and fin size on the rectangular prism with $D / H=0.2$ becomes large, the curve of the response amplitude shifts to that of the rectangular prism with $D / H=0.5$. The response amplitude of the rectangular prism with/without plate or fin is found to be related to the second moment of area of the prism.
\end{abstract}

\section{Keywords}

Flow-Induced Vibration, Cantilevered Rectangular Prism, Aspect Ratio, Stepped

Prism, Finned Prism, Response Amplitude, Resonant Reduced Velocity 


\section{Introduction}

Key features of a two-dimensional prism with a rectangular cross-section in a uniform flow are the generation of alternating vortices behind the prism and the separation bubble on the side surfaces of the prism. Flow characteristics of fluid forces and vortex shedding frequency have been dramatically changed by the side ratio of rectangular prisms [1]. Because of these features, the elastically mounted rectangular prisms experience the flow-induced vibration in crossflow directions by vortex-induced and galloping vibrations [2] [3]. The dynamic response of a prism depends on parameters such as the system damping and after body shape, that is, the structural part of a bluff body downstream from the flow separation points [4].

The flow around a finite-length prism without an end plate becomes a strongly three-dimensional structure [4]-[9]. There are distinct flow pattern changes along the prism height from the tip to the root. The near wake structure is dependent on the aspect ratio, $L / H$, of the prism, where $L$ is the length of the prism and $H$ is the height of the prism normal to the flow direction. From those investigations, the flow structure behind a finite prism is qualitatively identical to that of prisms above a critical aspect ratio, that is, $L / H \geq 5$. The wake structure is characterized by the trailing vortex from the tip of the prism and by the Kármán vortex shedding from the sides of the prism. On the other hand, below the critical aspect ratio, the arch and tip vortices can be observed in the wake flow of a finite prism.

The flow-induced vibration of a cantilever rectangular prism has been investigated by a few researchers [10] [11] [12]. Kiwata et al. [12] investigated the effect of side ratio on the response amplitude characteristic of cantilevered rectangular and $\mathrm{D}$-section prisms with an aspect ratio of 10 and a side ratio of less than $D / H=0.6$, where $D$ is the depth of a bluff body in the flow direction. However, it can be inferred that the length of the prism has non-negligible impacts on the flow-induced vibration features.

The wake flow behind a stepped circular cylinder, which consists of two circular cylinders with different diameters, was investigated to obtain the flow interaction in the wakes of two cylinders [13] [14]. The dynamics of an oscillating stepped cylinder have received less attention. On the other hand, the vortex-induced vibration of a tapered circular cylinder has been studied by several researchers [15] [16] [17]. Seyed-Aghazadeh et al. investigated the influence of the tapered ratio on the vortex-induced vibration of circular cylinders free to oscillate in the transverse direction. They found that the lock-in range of a circular cylinder with a small tapered ratio is sustained through largely reduced velocities [17].

The energy harvesting studies using piezoelectric materials and vortex-induced motions were reviewed by Sodano et al. [18] and Rostami and Armendei [19]. The flow-induced vibrational motion of columnar structures is a 
potential resource for the energy harvesting. The authors focused on the low-speed galloping vibration of the cantilever mounted rectangular prisms with a side ratio of $D / H \leq 0.5$ to develop a vibrational power generation system using the flow-induced vibration and an iron-gallium alloy [12] [20].

The objectives of the present study are to improve the increment and stability of the response amplitude and the decrement of the vibration's starting velocity by modifying the prism shape. The effects of length (aspect ratio) and the size of the additional structure (a plate and a fin) attached to the surface of a rectangular prism on the transverse vibration characteristics of cantilevered rectangular prisms with a side ratio of $D / H \leq 0.5$ have been investigated experimentally in a water tunnel.

\section{Experimental Apparatus and Methods}

\subsection{Water Tunnel and Test Models}

Figure 1 shows a schematic diagram of the test section of the water tunnel and measurement instruments. The experimental setup was similar to that as in [12] and hence will only be briefly described here. The experiment was performed in a water tunnel equipped with underground water. The rectangular test section of the water tunnel had a height of $400 \mathrm{~mm}$, a width of $167 \mathrm{~mm}$, and a length of 780 $\mathrm{mm}$. The prism was mounted elastically to a plate spring attached to the ceiling wall of the test section with a jig. Figure 2 shows the test models of rectangular prisms. Table 1 shows the specifications of the test models without additional structures. The prisms were made of stainless steel with smooth surfaces and had sharp edges. The rectangular prisms had a cross-section height $H$ of $20 \mathrm{~mm}$. The side ratio $D / H$ of the rectangular prism was changed 0.2 and 0.5 , where $D$ is the depth of the prism in the flow direction. The aspect ratio $L / H$ of the prisms was varied from 2.5 to 10 . Figure 3 shows side and cross-section views of a stepped rectangular prism that was joined to an additional plate with a thickness of $d=5$

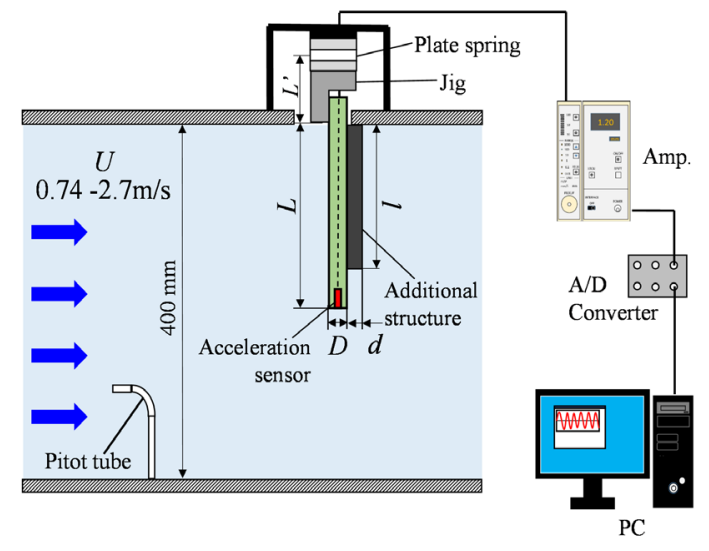

(a)

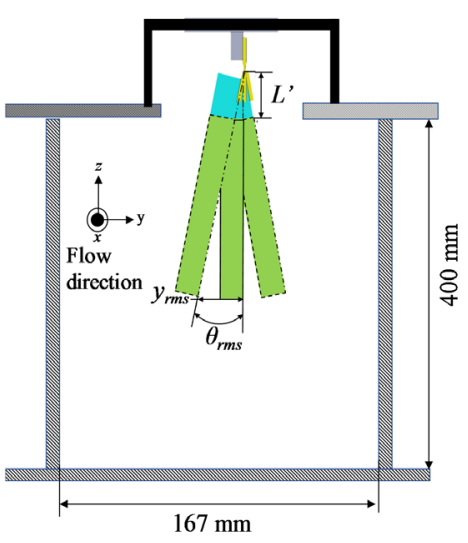

(b)

Figure 1. Test section of water tunnel and measurement instruments. (a) Side view; (b) Front view. 


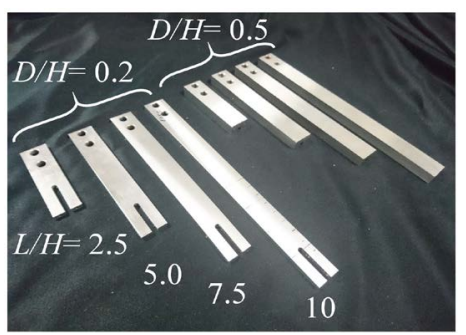

Figure 2. Overview of test models with different aspect ratio.

Table 1. Specification of test models without additional structures.

\begin{tabular}{|c|c|c|c|c|c|c|c|}
\hline \multirow{3}{*}{$L / H$} & \multirow{3}{*}{$\begin{array}{l}\text { Spring } \\
\text { thickness } \\
(\mathrm{mm})\end{array}$} & \multicolumn{6}{|c|}{$D / H$} \\
\hline & & \multicolumn{3}{|c|}{0.2} & \multicolumn{3}{|c|}{0.5} \\
\hline & & $f_{n}(\mathrm{~Hz})$ & $\delta$ & $C_{n}$ & $f_{n}(\mathrm{~Hz})$ & $\delta$ & $C_{n}$ \\
\hline \multirow{2}{*}{2.5} & 0.4 & 34.4 & 0.111 & 1.95 & 43.1 & 0.016 & 0.28 \\
\hline & 0.6 & 31.1 & 0.044 & 0.77 & 26.4 & 0.023 & 0.40 \\
\hline \multirow{3}{*}{5} & 0.4 & 23.8 & 0.035 & 0.61 & 15.1 & 0.036 & 0.63 \\
\hline & 0.6 & 38.7 & 0.026 & 0.46 & 24.8 & 0.026 & 0.46 \\
\hline & 0.8 & - & - & - & 33.9 & 0.017 & 0.30 \\
\hline \multirow{3}{*}{7.5} & 0.6 & 26.4 & 0.061 & 1.08 & 16.5 & 0.030 & 0.52 \\
\hline & 0.8 & 36.4 & 0.025 & 0.44 & 22.7 & 0.023 & 0.40 \\
\hline & 1.0 & 46.1 & 0.023 & 0.41 & 28.8 & 03017 & 0.30 \\
\hline \multirow{3}{*}{10} & 0.8 & 26.5 & 0.026 & 0.46 & 16.4 & 0.031 & 0.55 \\
\hline & 1.0 & 33.2 & 0.022 & 0.39 & 20.6 & 0.022 & 0.39 \\
\hline & 1.2 & 38.9 & 0.020 & 0.35 & 24.3 & 0.025 & 0.44 \\
\hline
\end{tabular}

$\mathrm{mm}$ and cross-section height of $h=20 \mathrm{~mm}$ to the front or back of the cantilevered rectangular prism with $D / H=0.2$ by screws. The stepped rectangular prism with the additional plate of different lengths was investigated to determine whether the stepped rectangular prism had hybrid vibration characteristics of the $D / H=0.2$ and 0.45 prisms. The length plate ratio $l / H$ was varied from 2.5 to 7.5. Figure 4 shows a cantilevered rectangular prism of $D / H=0.2$ with a fin which was fitted by an adhesive. The effects of the configuration of a fin fitted on the back of the cantilevered rectangular prisms to increase the flexural rigidity during flow-induced vibration were investigated. The acrylic resin fin spanned the entire length of the prism. The fin height to prism height ratio (height ratio) $1 / L$ was varied from 0.25 to 0.5 , and the fin depth to prism height ratio (depth ratio) $d / H$ was varied from 0.15 to 0.4 . The characteristic frequency $f_{c}$ of the prism with additional plate and fin increased from 0.3 to $3.7 \mathrm{~Hz}$ more than that without them. 


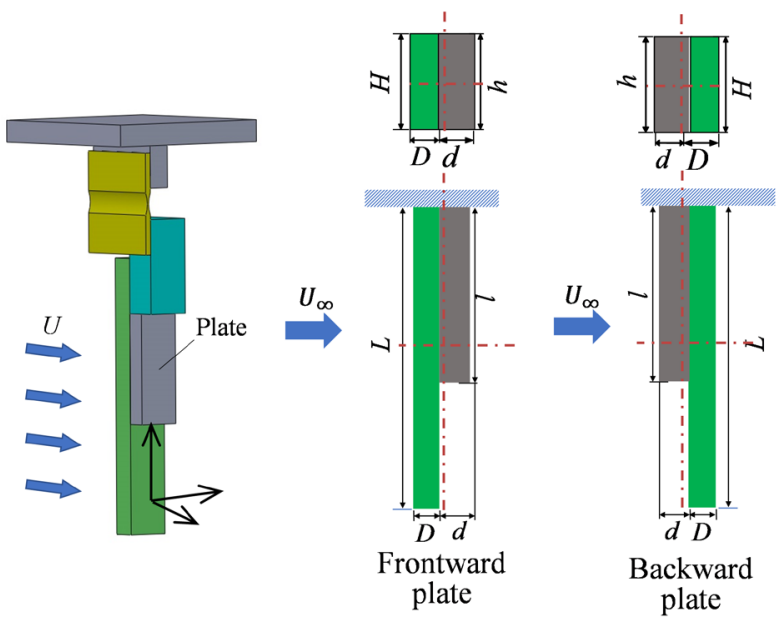

Figure 3. Cantilevered rectangular prism of $D / H=0.2$ with a plate $(h=20 \mathrm{~mm}, d=5 \mathrm{~mm}, 1 / H=2.5,5$ and 7.5$)$.

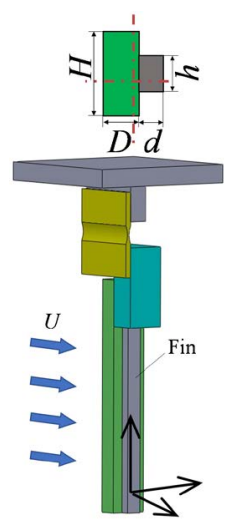

Figure 4. Cantilevered rectangular prism of $D / H=0.2$ with a fin $(h / H=0.25$ and $0.5, d / H=0.15-0.4)$.

\subsection{Experimental Method}

The uniform flow velocity $U$ was varied from 0.74 to $2.7 \mathrm{~m} / \mathrm{s}$ by controlling pump rotation speed and was measured using a pitot tube and digital differential pressure gauge (Nagano Keiki, GC50). The Reynold's number $\operatorname{Re}(=U H / \mathrm{v}$, where $V$ is the kinematic viscosity of water) range was $1.4 \times 10^{4}$ to $5.4 \times 10^{5}$. The reduced velocity $V_{r}\left(=U / f_{c} H\right)$ was calculated from the characteristic frequency of the prism $f_{c}$. Tip displacement $y$ was measured using an acceleration sensor (Showa Measuring Instrument, 2302CW) implanted inside the tip of the prism and an integrator (RION UV-12 and UV-05). The signal output of the integrator was converted using a 12-bit A/D converter with a sampling frequency of $2 \mathrm{kHz}$, and 10,000 data points were recorded. The characteristic frequency of test models $f_{c}$ was measured using the FFT analyzer (ONO SOKKI, CF-5201). The characteristic frequency $f_{\mathrm{c}}$ of the prism was set in a constant value from $16.4 \mathrm{~Hz}$ to $38.9 \mathrm{~Hz}$ using different thicknesses of plate springs. The root-mean-square (RMS) values of the fluctuation of displacement of a prism tip $y_{\mathrm{rms}}$, the non-dimensional displacement amplitude $\eta_{\mathrm{rms}}\left(=y_{\mathrm{rms}} / H\right)$, and the angle ampli- 
tude $\theta_{\mathrm{rms}}\left[\approx \tan ^{-1}\left\{y_{\mathrm{rms}} /(L+L)\right\} \cdot 180 / \pi\right.$, where $L$ 'is length of a jig] were calculated by using a personal computer. In order to prevent breakage of the plate spring, the maximum value of the prism amplitude was limited to $\eta_{\mathrm{rms}} \approx 0.2$. The reduced mass damping $C n(=2 m \delta / \rho D H$, where $m, \delta$, and $\rho$ are the mass per unit length of the system, the logarithmic decrement of the structural damping parameter of a prism, and the water density, respectively) was measured by considering the initial displacement obtained by hitting the prism with a hammer in stationary water.

\section{Experimental Results and Discussion}

\subsection{Effect of Aspect Ratios of the Rectangular Prism}

Figure 5 shows the non-dimensional displacement amplitude $\eta_{\mathrm{rms}}$ of a cantilevered rectangular prism with an aspect ratio of $L / H=10$ for different natural frequencies using different thicknesses of plate springs. After the vibration begins, the amplitude $\eta_{\mathrm{rms}}$ increases linearly with the reduced velocity $V r$. Although the velocity in this experiment is a different range of Reynolds number, each curve of the non-dimensional displacement amplitude $\eta_{\text {rms }}$ has good agreement. The reduced velocity of the vibration onset and the non-dimensional displacement amplitudes do not depend on the test models' characteristic frequency in still water, $f_{\mathrm{c}}$. The onset vibration of rectangular prisms with $D / H=0.2$ and 0.5 occurred near $V_{r} \approx 1.5$ and 3.0, respectively. Thus, the reduced velocity of the vibration onset for prisms with the small side ratio of $D / H=0.2$ is lower than that for the large side ratio of $D / H=0.5$. The reduced resonance velocities for the rectangular prisms with side ratios of $D / H=0.5$ and 0.2 were $V r_{\text {cr }} \approx 8.0$ and 7.3 [21] [22]. This is evidence that the rectangular prisms with $D / H=0.5$ and 0.2 behave as the low-speed galloping vibration at a reduced velocity that is lower than the resonant reduced velocity $V_{\text {rr }}$.

The effect of aspect ratio on the nondimensional displacement amplitude $\eta_{\mathrm{rms}}$ and the ratio of the actual vibration frequency of the prism in a flow on the

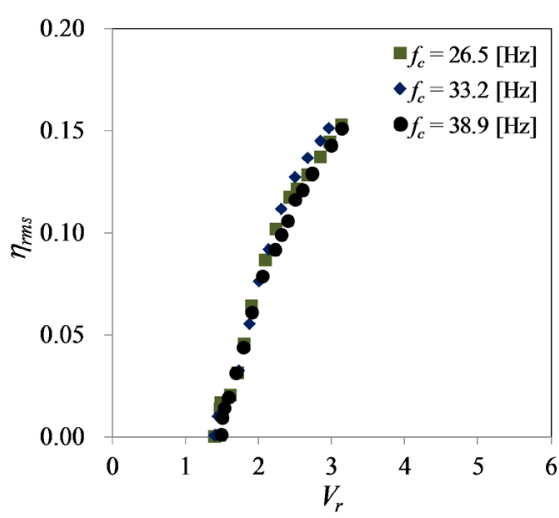

(a)

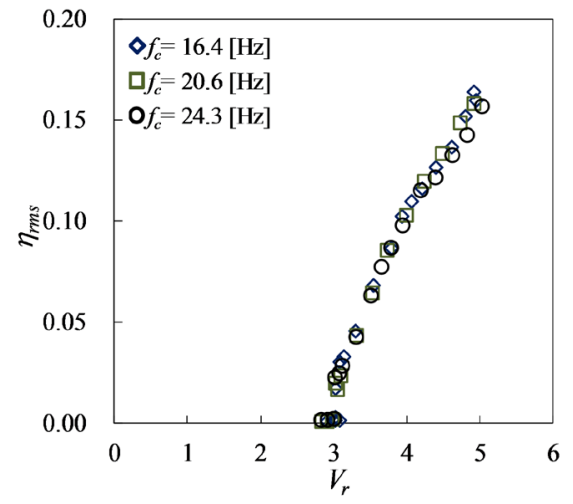

(b)

Figure 5. Response amplitude of a cantilevered rectangular prism with an aspect ratio of $L / H=10$ for different natural frequencies. 
characteristic frequency of the still water, $f / f_{\mathcal{O}}$, is shown in Figure 6 . The vibration onset of rectangular prisms does not completely depend on the aspect ratio $L / H$. After the vibration begins, the nondimensional displacement amplitude $\eta_{\text {rms }}$ increases with an uptick in the reduced velocity $V_{r}$. The frequency ratio $f / f_{c}$ decreases slightly with an increase in the reduced velocity $V_{r}$. It seems that the vibration frequency in a fluid depends on the vibration amplitude. Namely, it is affected by the added (virtual) mass and viscosity. Figure 7 shows the nondimensional increment rate of the response amplitude $\mathrm{d} \eta_{\mathrm{rms}} / \mathrm{d} V_{r}$ of prisms from the onset of vibration to the end of measurement. The value of $\mathrm{d} \eta_{\mathrm{rms}} / \mathrm{d} V_{r}$ rises with an increase in the aspect ratio $L / H$. The increment rate of a rectangular prism with $D / H=0.2$ is larger than that with $D / H=0.5$ for $2.5 \leq L / H<10$. For the rectangular prisms with $D / H=0.2$, the increment rate keeps constant over $L / H=7.5$.

The displacement amplitude $\eta_{\mathrm{rms}}$ as converted into the angle of amplitude $\theta_{\mathrm{rms}}$ is shown in Figure 8. After the vibration begins, the angle of amplitude $\theta_{\text {rms }}$ also increases linearly with the reduced velocity $V_{r}$. The curves of the amplitude angle of the prisms with aspect ratios of $L / H=7.5$ and 5 are in good agreement with those that have a large aspect ratio of $L / H=10$. Although the displacement amplitude of the prism tip does not have the same displacement $y_{\mathrm{rms}}$ as shown in Figure 6, the angle of amplitude $\theta_{\mathrm{rms}}$ is similar to that of the rectangular prisms with aspect ratios of $L / H \geq 5$. However, the amplitude angle of the prisms with aspect ratio of $L / H=2.5$ is smaller than that of those with an aspect ratio of $L / H$ $\geq 5$. The distinct difference in the vibration characteristic appears on a prism with a small aspect ratio of 2.5. It has a connection with the flow structure behind a finite prism with a critical aspect ratio, that is, $L / H \geq 5$ [5].

Figure 9 and Figure 10 show the time lapse of the tip displacement $y$ for a rectangular prism with $D / H=0.2$ and aspect ratios of $L / H=2.5$ and 10 . For $L / H$ $=2.5$, the vibration is not stable for $V_{r}=1.75$ and 3.2. On the other hand, for $L / H=10$, the amplitude of the vibration has a uniform amplitude for $V_{r}=2.0$ and 3.15. These phenomena are causally related to the two-dimensionality of the

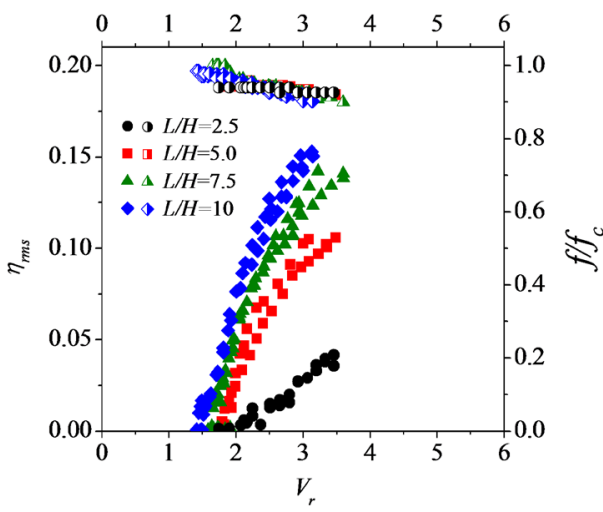

(a)

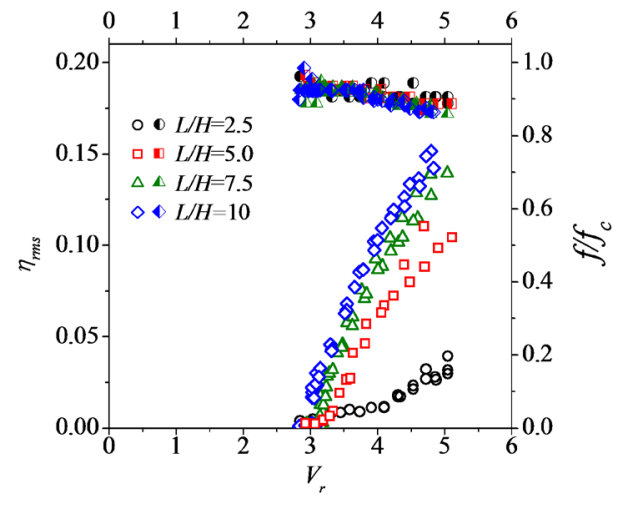

(b)

Figure 6. Response amplitude and normalized frequency of cantilevered rectangular prisms with different aspect ratios. (a) $D / H=0.2$; (b) $D / H=0.5$. 


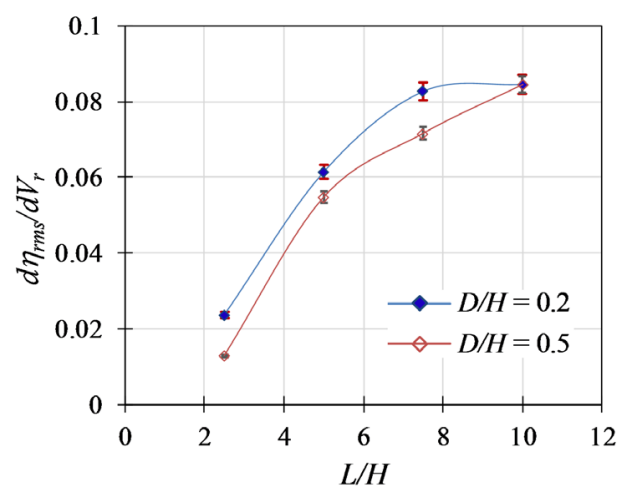

Figure 7. Non-dimensional increment rate of response amplitude $d \eta_{r m s} / d V_{r}$ of prisms.

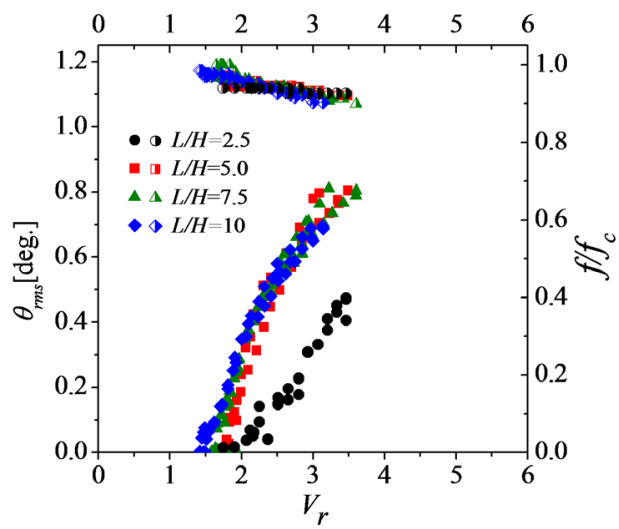

(a)

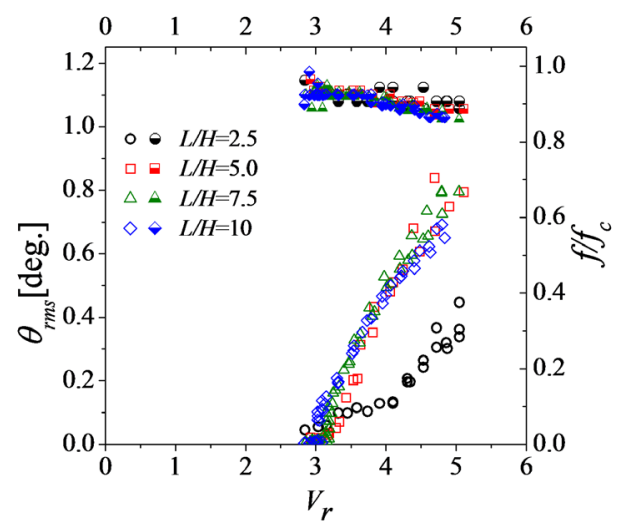

(b)

Figure 8. Response angle amplitude of cantilevered rectangular prisms with different aspect ratios. (a) $D / H=0.2$; (b) $D / H=0.5$.

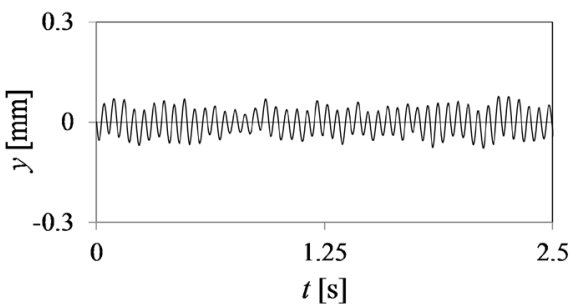

(a)

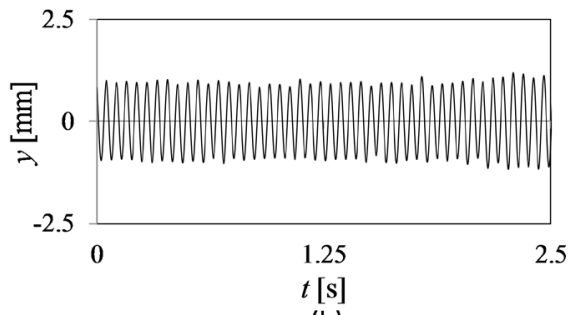

(b)

Figure 9. Tip displacement over time for a rectangular prism with $D / H=0.2$ and $L / H=2.5$. (a) $V_{r}=1.74(U$ $=1.34 \mathrm{~m} / \mathrm{s})$; (b) $V_{r}=3.2(U=2.46 \mathrm{~m} / \mathrm{s})$. 


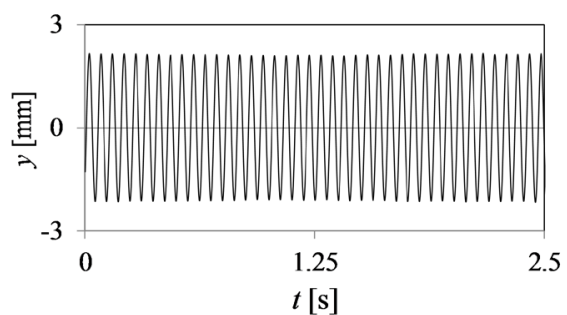

(a)

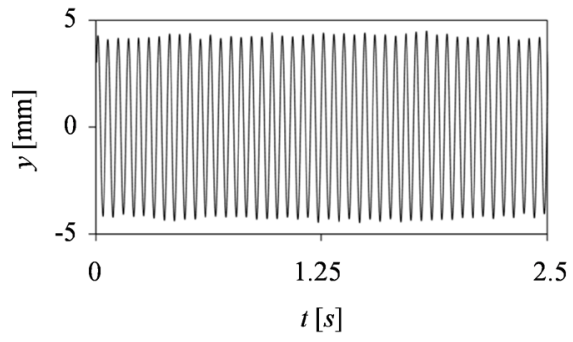

(b)

Figure 10. Tip displacement over time for a rectangular prism with $D / H=0.2$ and $L / H=10$. (a) $V_{r}=2.0(U$ $=1.33 \mathrm{~m} / \mathrm{s})$; (b) $V_{r}=3.15(U=2.45 \mathrm{~m} / \mathrm{s})$.

flow structure of the wake, that is, the interaction between the lengthwise vortices and a tip vortex, in which the Kármán vortex shedding is suppressed and replaced with arch vortex formation for the small aspect ratio [5] [6] [7] [8] [9].

The time lapses of the tip displacement $y$ for a rectangular prism of $D / H=0.5$ with aspect ratios of $L / H=2.5$ and 10 are shown in Figure 11 and Figure 12. In the case of a rectangular prism with $D / H=0.5$, the amplitude of the vibration for a large aspect ratio of $L / H=10$ did not even out, and its stability is the same as that for a small aspect ratio of $L / H=2.5$. It seems that the unstable waveform is generated by the irregular flow reattachment on the side wall of the rectangular prism due to the vibration [1] [22].

To evaluate the stability of a vibration, the variation in the nondimensional standard deviation of the peak displacement for a rectangular prism $p_{\theta \mathrm{rms}} / \theta_{\mathrm{rms}}$ with respect to the reduced velocity $V_{r}$ is shown in Figure 13 . Here, $p_{\theta r m s}$ is the root-mean-square (RMS) of the value of subtracting $p_{\text {tave }}$ from the absolute value of the peak displacement $p_{\theta \mathrm{i}}$ for the waveform, and $p_{\text {धave }}$ is the time average of $\left|p_{\theta i}\right|$. These values are given by the following equations:

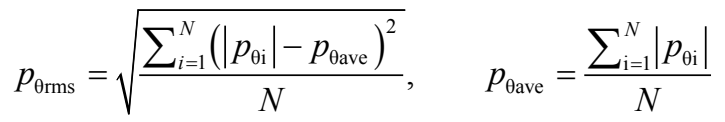

where $N$ is the number of waveform peaks [12]. For a slightly reduced velocity and the small amplitude of the vibration, the nondimensional standard deviations of the peak displacements $p_{\theta \mathrm{rms}} / \theta_{\mathrm{rms}}$ are large. For a largely reduced velocity, the $p_{\theta \mathrm{rms}} / \theta_{\mathrm{rms}}$ of the rectangular prisms with a small aspect ratio of $L / H=2.5$ are larger than for those with a large aspect ratio. The stability of the vibration for a prism with a side ratio of $D / H=0.2$ and an aspect ratio of $L / H \geq 5$ tends to 


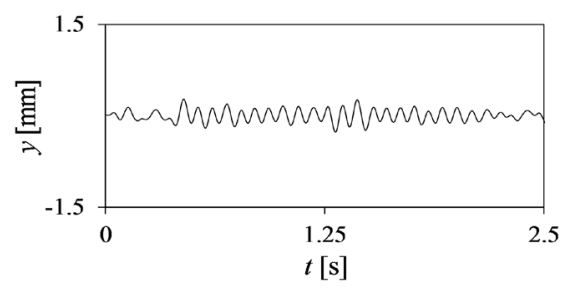

(a)

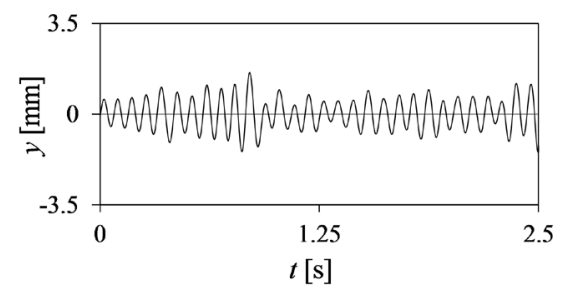

(b)

Figure 11. Tip displacement over time for a rectangular prism with $D / H=0.5$ and $L / H=2.5$. (a) $V_{r}=3.03$ $(U=1.60 \mathrm{~m} / \mathrm{s})$; (b) $V_{r}=4.72(U=2.49 \mathrm{~m} / \mathrm{s})$.

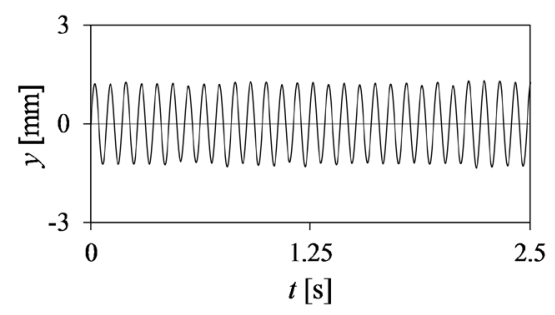

(a)

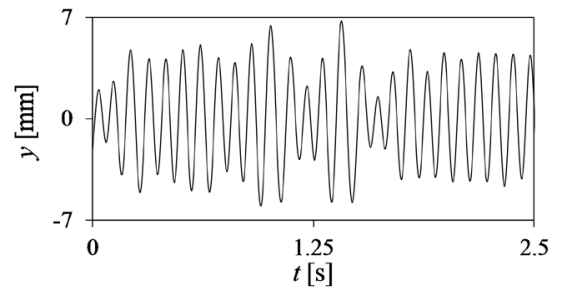

(b)

Figure 12. Tip displacement over time for a rectangular prism with $D / H=0.5$ and $L / H=10$. (a) $V_{r}=3.09$ $(U=1.61 \mathrm{~m} / \mathrm{s})$; (b) $V_{r}=5.04(U=2.45 \mathrm{~m} / \mathrm{s})$.

increase relatively more than that for the other prisms.

\subsection{Effect of Added Plate Length}

The effect of the configurations of additional structures, that is, a plate as shown in Figure 3 on the flow-induced vibration of a rectangular prism with $D / H=0.2$ was investigated. If the prism was a flexible material, the rectangular prism was bent by the drag force. An additional structure made of an acrylic resin was fitted to the front or back of the rectangular prism in order to prevent it from bending. Furthermore, the response amplitude of the vibration for the prism with an additional structure might have characteristics of rectangular prisms with both 


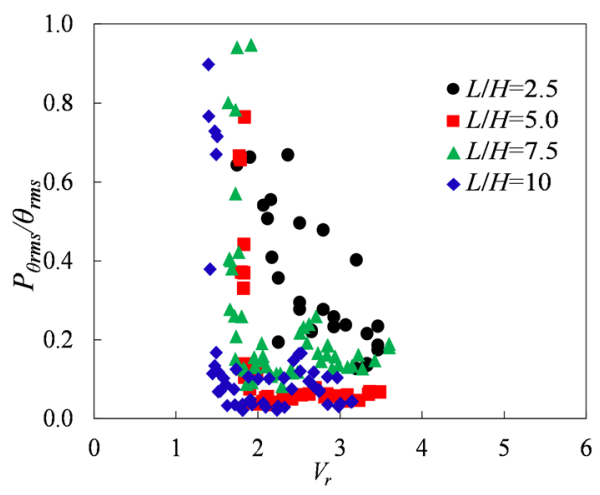

(a)

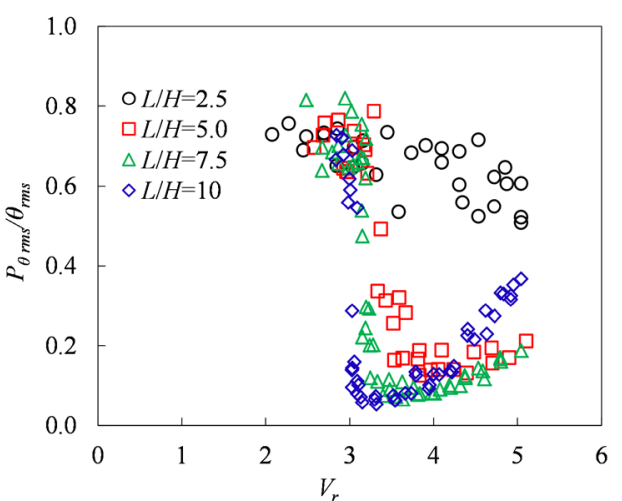

(b)

Figure 13. Non-dimensional standard deviation of peak displacement for a rectangular prism of $D / H=0.2$ and 0.5 with respect reduced velocity $V_{r^{r}}$ (a) $D / H=0.2$; (b) $D / H=0.5$.

$D / H=0.2$ and 0.45 .

Figure 14 shows the nondimensional displacement amplitude $\eta_{\mathrm{rms}}$ of a cantilevered rectangular prism with a side ratio of $D / H=0.2$ and an aspect ratio of $L / H=10$ for different lengths of plates. In the case of the added plate on the front surface of a rectangular prism with $1 / H=2.5$ (as shown in Figure 14(a)), the reduced velocity of the vibration onset increased slightly more than for the rectangular prism without a plate. For $1 / H=5.0$ and 7.5 , the reduced velocity of the vibration onset rose with an increase in the length of the plate $1 / H$. The increment rate of the response amplitude of a rectangular prism with $l / H=2.5$ is the same as that for the other lengths of plates. The increment rate of the response amplitude of a rectangular prism with $1 / H=7.5$ is larger than that of $D / H$ $=0.5$ without a plate.

As shown in Figure 14(b), in the case of the added plate on the back surface of a rectangular prism, the characteristics were similar to the case of the added plate on the front surface of a rectangular prism. Therefore, if the length of the added plate was increased, the response amplitude of a cantilevered rectangular prism with $D / H=0.2$ only shift to that of a cantilevered rectangular prism with $D / H=0.5$. The rectangular prism with an added plate does not have a hybrid response amplitude, that is, the characteristics for a large response amplitude to the largely reduced velocity.

Figure 15 and Figure 16 show the time lapses for tip displacement $y$ and the nondimensional standard deviation of peak displacement $p_{\text {yrms }} / y_{\mathrm{rms}}$ for a rectangular prism of $D / H=0.2$ with an added plate. The stability of the vibration of a rectangular prism with an added plate is not good. Because the vortex structure shedding from the rectangular prism with an added plate depends on the side ratio [1] [22], it changes along the length of the rectangular prism like the stepped circular cylinder [13]. Therefore, the $p_{\text {yrms }} / y_{\mathrm{rms}}$ of a rectangular prism of $D / H=0.2$ with an added plate was larger than that of $D / H=0.2$ without an added plate at the same reduced velocity. The characteristics shifted to those of a rectangular prism with $D / H=0.5$. 


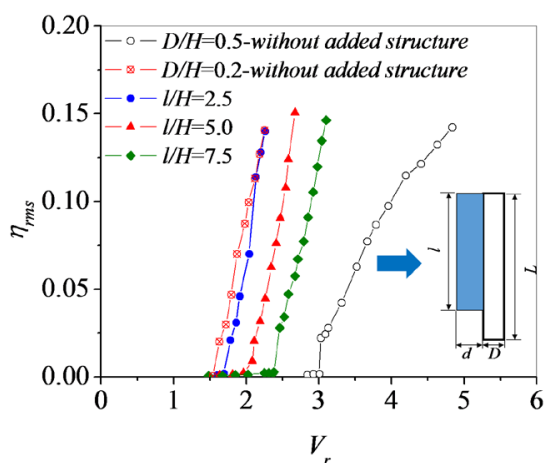

(a)

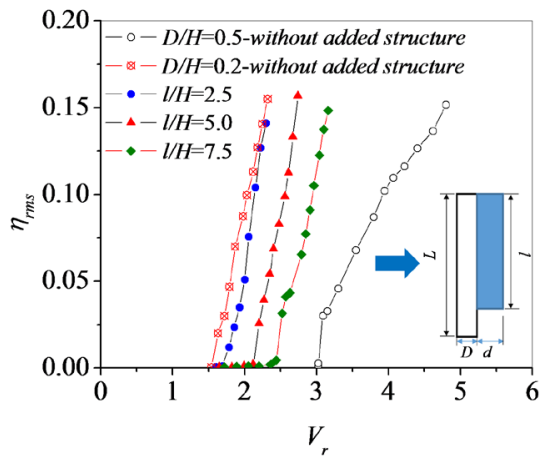

(b)

Figure 14. Response amplitude of cantilevered rectangular prism with a plate $(D / H=$ 0.2). (a) Plateput on the front surface; (b) Plateput on the back surface.

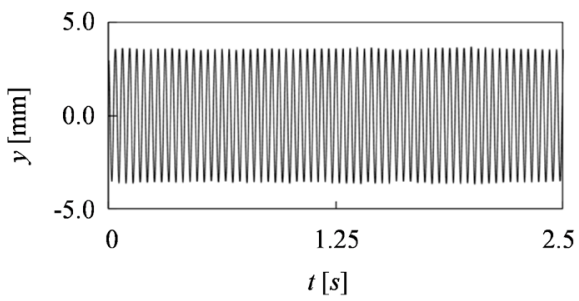

(a) $l / H=2.5(V r=2.22, U=1.21 \mathrm{~m} / \mathrm{s})$

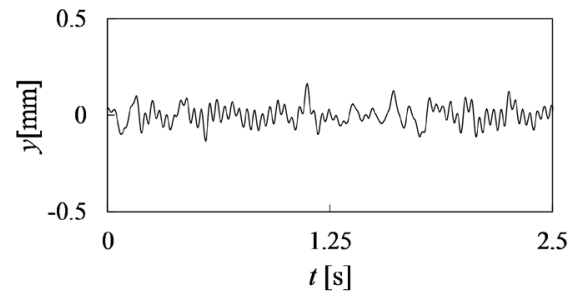

(c) $l / H=7.5(V r=2.38, U=1.24 \mathrm{~m} / \mathrm{s})$

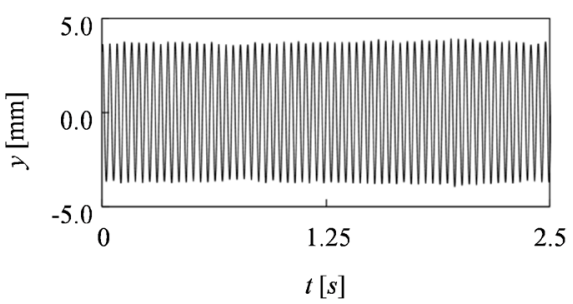

(b) $l / H=5.0(V r=2.67, U=1.42 \mathrm{~m} / \mathrm{s})$

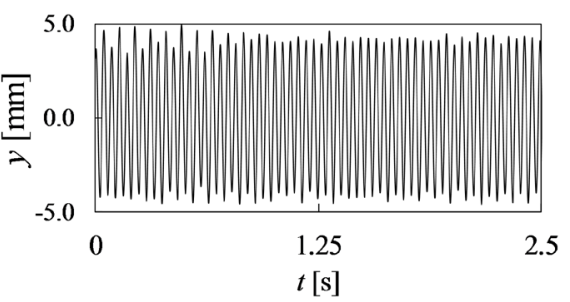

(c) $l / H=7.5(V r=3.16, U=1.62 \mathrm{~m} / \mathrm{s})$

Figure 15. Time histories tip displacement for a rectangular prism of $D / H=0.2$ with an added plate on the front surface.

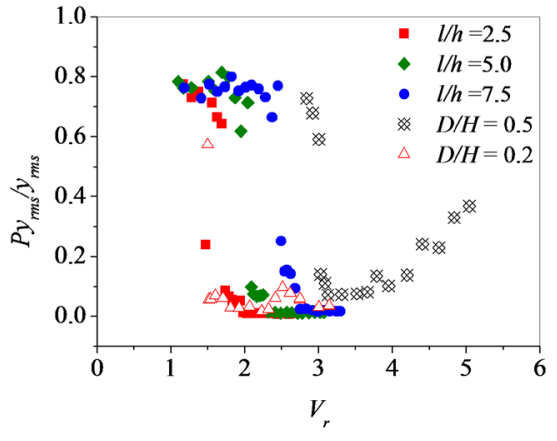

(a)

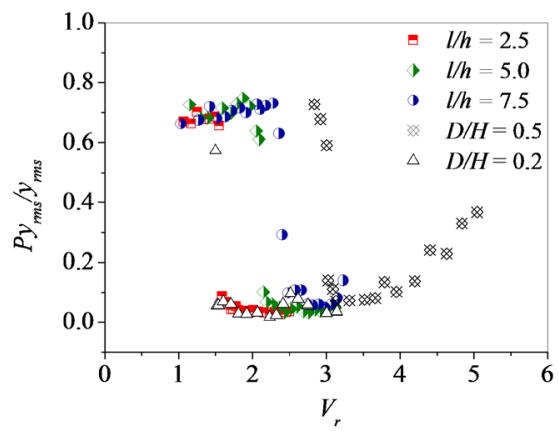

(b)

Figure 16. Non-dimensional standard deviation of peak displacement for a rectangular prism of $D / H=0.2$ with an added plate. (a) Plate put on the front surface; (b) Plate put on the back surface.

The relationship between the response amplitude and the flexural rigidity of 
the stepped rectangular prism was examined. Figure 17 shows variation of second moment of area $I_{1}$ with the reduced velocities at the $15 \%$ non-dimensional response amplitude of a stepped rectangular prism $V r_{0.15}$. The second moment area $I_{1}\left(=I_{z}\right)$ of the stepped rectangular prism with respect to $z$-axis is given in Equation (2).

$$
\begin{aligned}
& I_{1}\left(=I_{z}\right)=\frac{l(D+d)^{3}+(L-l) D^{3}}{3}-\{l(D+d)+(L-l) D\} e_{1}^{3} \\
& e_{1}=0.5 \frac{l(D+d)^{2}+(L-l) D^{2}}{l(D+d)+(L-l) D}
\end{aligned}
$$

The second moment of area $I_{1}$, that is the rigidity of prism, increased with increasing length of additional plate. Although the rigidity of prism increased by the plate-type additional structure, the reduced velocity at the $15 \%$ non-dimensional response amplitude of a stepped rectangular prism $V r_{0.15}$ did not decrease. The plate-type additional structure does not give a good effect on the vibration characteristics, that is stable amplitude and decrease in velocity of onset vibration. The effect of fin-type additional structure on the response amplitude is described in the following section.

\subsection{Effect of Added Fin Size}

The effect of the configuration of a fin, which was fitted to the back of a rectangular prism, on flow-induced vibration of a rectangular prism with $D / H=0.2$ was investigated. The nondimensional displacement amplitude $\eta_{\mathrm{rms}}$ of a cantilevered rectangular prism with a side ratio of $D / H=0.2$ and an aspect ratio of $L / H$ $=10$ for different sizes of fins are shown in Figure 18. The response amplitude $\eta_{\mathrm{rms}}$ increased linearly with an uptick in the reduced velocity $V_{r}$, which was similar to the response amplitude of the rectangular prism without a fin. The reduced velocity of the vibration onset went up with an increase in the depth ratio of the fin, $d / H$. The increment rate of the nondimensional response amplitude

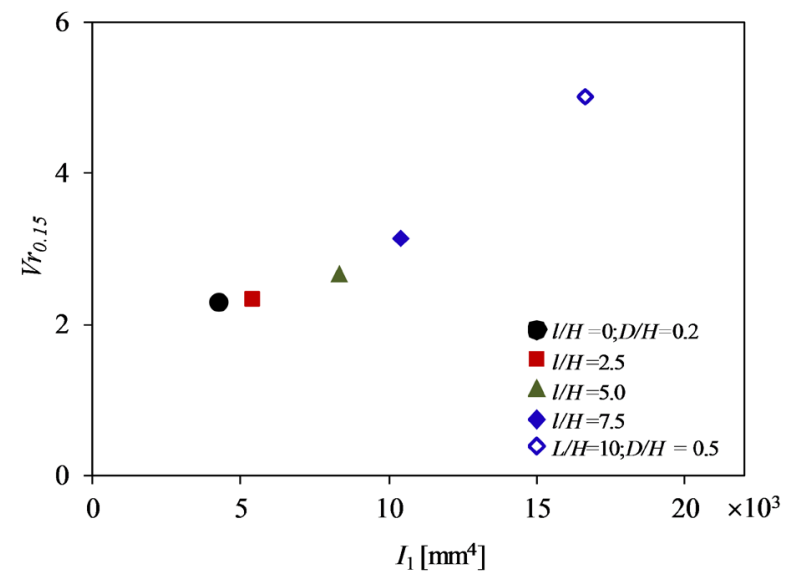

Figure 17. Variation of the second moment area $I$ with the reduced velocity at $15 \%$ non-dimensional response amplitude of a stepped rectangular prism $V_{t 0.15}$. 


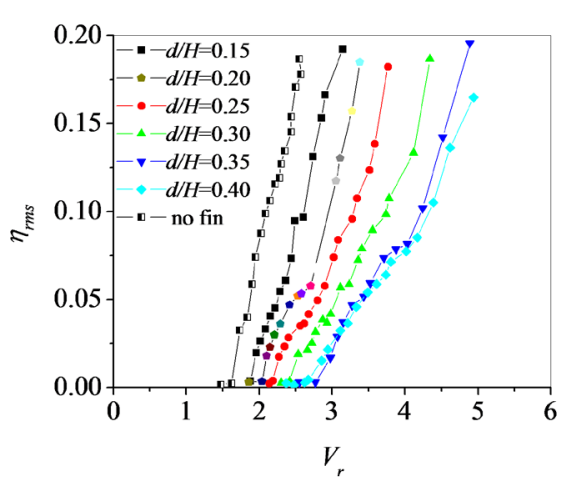

(a)

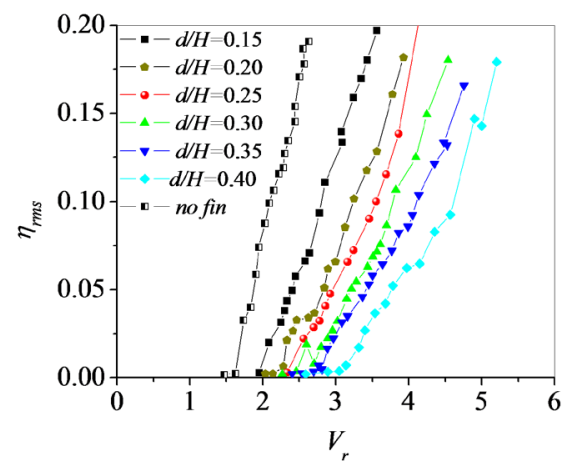

(b)

Figure 18. Response amplitude of cantilevered rectangular prism of $D / H=0.2$ with a fin. (a) $h / H=0.25$; (b) $h / H=0.50$.

$\eta_{\text {rms }}$ for the reduced velocity $V r$ of a rectangular prism without a fin was higher than that of a prism with a fin. The increment rate $\mathrm{d} \eta_{\mathrm{rms}} / \mathrm{d} V r$ of the prism with a fin of $d / H=0.35$ and 0.4 is low in the region of initial vibration.

Figure 19 shows the variation with time of the tip displacement of a rectangular prism of $D / H=0.2$ with a fin of $(h / H, d / H)=(0.5,0.4)$. These vibrations do not have a uniform amplitude for each reduced velocity, similar to the rectangular prism with $D / H=0.5$ without a fin as shown in Figure 12. Figure 20 shows the variation of the nondimensional standard deviation of peak displacement of a rectangular prism $p_{\text {yms }} / y_{\mathrm{rms}}$ with respect to the reduced velocity $V r$. The values of $p_{\text {yrms }} / y_{\mathrm{rms}}$ of the rectangular prism with a fin are larger than those of the rectangular prism without a fin. The variation with time of the tip displacement of a rectangular prism of $D / H=0.2$ with a fin, shown in Figure 19(b), is similar to that of a rectangular prism of $D / H=0.5$ without a fin, shown in Figure 12(b). The stable flow-induced vibration was prevented by the fin and increased the stiffness of the thin rectangular prism.

Therefore, the relationship between the response amplitude and the flexural rigidity of the rectangular prism with a fin was examined. The second moment of area indicates the flexural rigidity of the rectangular prism. The second moment of area of the rectangular $\operatorname{prism} I_{2}$ with respect to $y$-axis with a fin is given in Equation (3).

$$
I_{2}\left(=I_{y}\right)=\frac{H D^{3}+h d^{3}}{3}+h d D(D+d)-\left\{\frac{h d\left(D+\frac{d}{2}\right)+\frac{H D^{2}}{2}}{h d+H D}\right\}^{2}(H d+h d)
$$

where the quantities are defined in the nomenclature section. For example, the values of the second moment of area of a rectangular prism with $D / H=0.2$ with and without a fin $(h / H, d / H)=(0.25,0.15)$ were $273 \mathrm{~mm}^{4}$ and $107 \mathrm{~mm}^{4}$, respectively. The flexural rigidity of a rectangular prism with a fin is 2.6 times that of a rectangular prism without a fin.

The response amplitude $\eta_{\mathrm{rms}}$ of the second moment of area of the rectangular 


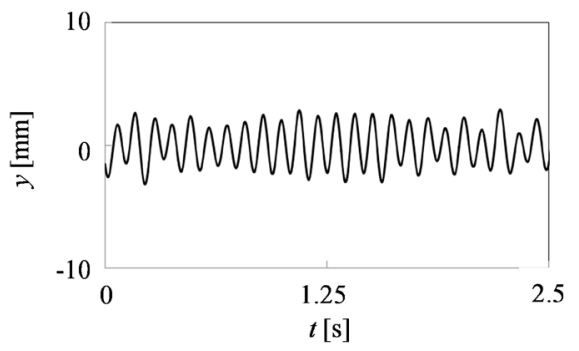

(a)

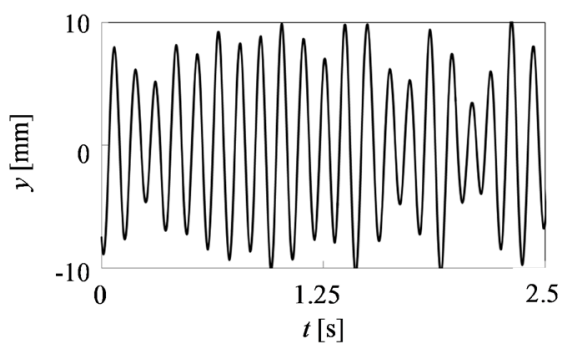

(b)

Figure 19. Variation with time of the tip displacement of the rectangular prism of $D / H=$ 0.2 with a fin of $(h / H, d / H)=(0.5,0.4)$. (a) $V_{r}=4.35(U=1.70 \mathrm{~m} / \mathrm{s}) ;(\mathrm{b}) V_{r}=5.73(U=$ $1.96 \mathrm{~m} / \mathrm{s})$.

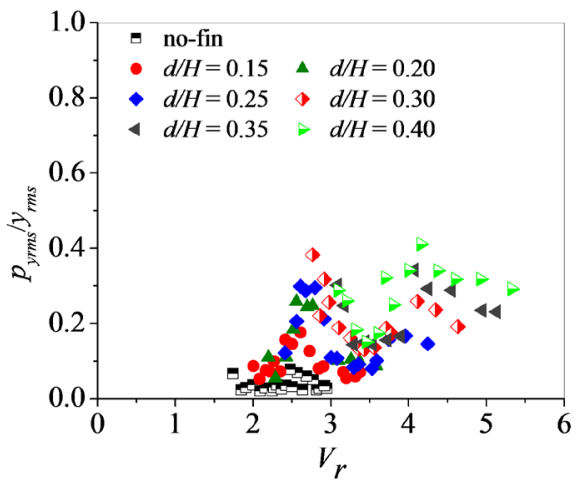

(a)

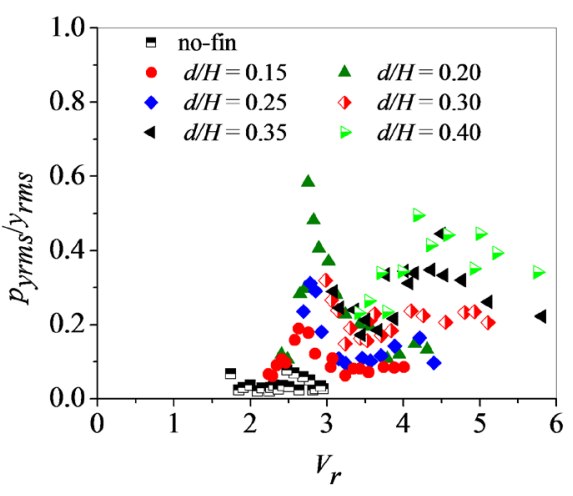

(b)

Figure 20. Non-dimensional standard deviation of peak displacement of a rectangular prism of $D / H=0.2$ with a fin $p_{\text {yrms }} / y_{\text {rms }}$ with respect to the reduced velocity $V_{r}$ (a) $h / H=$ 0.25 ; (b) $h / H=0.50$.

prisms with $(D / H, h / H, d / H)=(0.3,0,0),(0.2,0.5,0.15)$, and $(0.2,0.25,0.2)$ are shown in Figure 21. The second moments of cross-section area had similar values, that is, $I_{2}=360$ to $396 \mathrm{~mm}^{4}$. The curve of the response amplitude of the rectangular prism with a fin was in good agreement with that of the rectangular prism without a fin. Therefore, the reduced velocities at the $15 \%$ nondimensional response amplitude of a rectangular prism, $V r_{0.15}$, were plotted with respect to the second moment of cross-section area $I$ of rectangular prisms with $D / H=0.1$ to 0.5 in Figure 21 [12]. The experimental results indicate that the response amplitude of a rectangular prism depends on the second moment area of a rectangular prism. Further research on the wake structure of rectangular prism would clarify the relationship between the free-vibration characteristics, the fin shape, and the flexural rigidity of the rectangular prism.

\section{Conclusions}

The experiment on vibration characteristics of rectangular prisms with different aspect ratios and additional structures has been investigated in a water tunnel. The main conclusions of the present study are as follows:

1) The reduced velocity of the vibration onset and the increment rate of the 


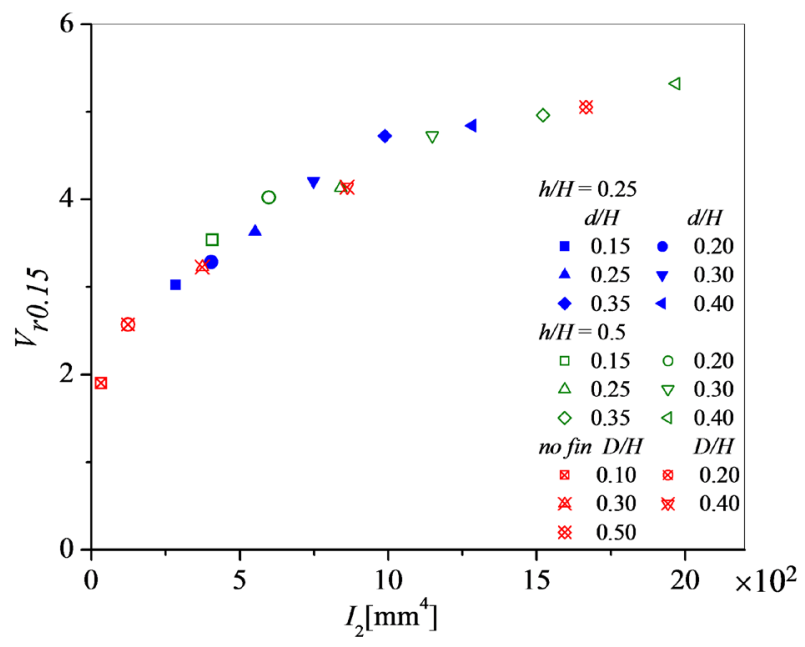

Figure 21. Variation of second moment of area $I_{2}$ with the reduced velocity at $15 \%$ non-dimensional response amplitude of a rectangular prism $V r_{0.15}$.

response amplitude of displacement are influenced by the aspect ratio of $L / H$. The vibration characteristics of angle amplitude of the prisms with aspect ratio $L / H \geq 5.0$ are not similar to those with aspect ratios below the critical aspect of $L / H=2.5$. The prisms with an aspect ratio of $L / H=2.5$ have nonuniform amplitude, small angle amplitude, and a low increment rate of the response amplitude. The prisms with $L / H=10$ have stable vibration and a high increment rate of the response amplitude.

2) In the case of a rectangular prism with a plate, the reduced velocity of the initial vibration increases as the length of a plate increases. The values of the nondimensional standard deviation of the peak displacement of a rectangular prism with a plate are larger than those of a rectangular prism without a plate.

3) In the case of a rectangular prism with a fin, the reduced velocity of the initial vibration increases as the depth ratio of a fin increases. The values of the nondimensional standard deviation of the peak displacement of a rectangular prism with a fin are larger than those of a rectangular prism without a fin. The response amplitude of a rectangular prism depends on the second moment of area of the rectangular prism, independent of whether the prism has a fin.

\section{Acknowledgements}

This work was supported by JST CREST Grant JPMJCR15Q1 (15665024). The authors are thankful to Professor Emeritus Dr. Okajima for productive discussions and to technician Mr. Kuratani and students Mr. Yamaguchi, Mr. Mizukami and Mr. Nagase for their help with the experiment.

\section{Conflicts of Interest}

The authors declare no conflicts of interest regarding the publication of this paper. 


\section{References}

[1] Okajima, A. (1982) Strouhal Numbers of Rectangular Cylinders. Journal of Fluid Mechanics, 123, 379-398. https://doi.org/10.1017/S0022112082003115

[2] Naudascher, E. and Rockwell, D. (2005) Flow-Induced Vibrations: An Engineering Guide. Dover Publications, Inc., Mineola.

[3] Garrett, J.L. (2003) Flow-Induced Vibration of Elastically Supported Rectangular Cylinders. Doctor Thesis, Iowa State University, Ames.

[4] Parkinson, G. (1989) Phenomena and Modelling of Flow-Induced Vibrations of Bluff Bodies. Progress in Aerospace Sciences, 26, 169-224.

https://doi.org/10.1016/0376-0421(89)90008-0

[5] Sumner, D. (2013) Flow above the Free End of a Surface-Mounted Finite-Height Circular Cylinder: A Review. Journal of Fluids and Structures, 43, 41-63. https://doi.org/10.1016/j.jfluidstructs.2013.08.007

[6] Kawamura, T., Hiwada, M., Hibino, T. and Mabuchi, I. (1984) Flow around a Finite Circular Cylinder on a Flat Plate Cylinder. Bulletin of JSME, 27, 2142-2151. https://doi.org/10.1299/jsme1958.27.2142

[7] Tanaka, S. and Murata, S. (1999) An Investigation of the Wake Structure and Aerodynamic Characteristics of a Finite Circular Cylinder. JSME International Journal, 42, 178-187. https://doi.org/10.1299/jsmeb.42.178

[8] Wang, H.F., Zhou, Y., Chan, C.K., Wong, W.O. and Lam, K.S. (2004) Flow Structure around a Finite-Length Square Prism. Proc. 15th Australasian Fluid Mechanics Conference, Sydney, 13-17 December 2004, 1-4. http://web.aeromech.usyd.edu.au/15afmc/proceedings/papers/AFMC00189.pdf

[9] Wang, H.F. and Zhou, Y. (2009) The Finite-Length Square Cylinder near Wake. Journal of Fluid Mechanics, 638, 453-490. https://doi.org/10.1017/S0022112009990693

[10] Hu, G., Tse, K.T. and Kwok, K.C.S. (2015) Galloping of Forward and Backward Inclined Slender Square Cylinders. Journal of Wind Engineering and Industrial Aerodynamics, 142, 232-245. https://doi.org/10.1016/j.jweia.2015.04.010

[11] Ishikawa, H., Yamada, S. and Ichikawa, S. (2012) Unsteady Vortex Structures in the Wake behind Bluff Bodies. Journal of Fluid Science and Technology, 7, 224-230. https://www.jstage.jst.go.jp/article/jfst/7/2/7_224/_pdf https://doi.org/10.1299/jfst.7.224

[12] Kiwata, T., Yamaguchi, M., Kono, T. and Ueno, T. (2014) Water Tunnel Experiments on Transverse-Galloping of Cantilevered Rectangular and D-Section Prisms. Journal of Fluid Science and Technology, 9, 1-5. https://doi.org/10.1299/jfst.2014jfst0056

[13] Ko, N.W.M., Leung, W.L. and Au, H. (1982) Flow Behind Two Coaxial Circular Cylinders. Journal of Fluids Engineering, 104, 223-227. https://doi.org/10.1115/1.3241814

[14] Wu, P., Hanu, H. and Miyakoshi, K. (2007) Generation of New Frequencies by Nonlinear Interaction between Different Vortex Shedding Frequencies in Span Wise Direction of a Stepped Circular Cylinder. Transactions of the Japan Society of Mechanical Engineers B, 73, 103-111. (In Japanese) https://www.jstage.jst.go.jp/article/kikaib1979/73/725/73_725_103/_pdf/-char/ja https://doi.org/10.1299/kikaib.73.103

[15] Balasubramanian, S., Haan, F.L., Szewczyk, A.A. and Skop, R.A. (2001) An Experimental Investigation of the Vortex-Excited Vibrations of Pivoted Tapered Circular 
Cylinders in Uniform and Shear Flow. Journal of Wind Engineering and Industrial Aerodynamics, 89, 757-784. https://doi.org/10.1016/S0167-6105(00)00093-3

[16] Zeinoddini, M., Tamimi, V. and Seif, M.S. (2013) Stream-Wise and Cross-Flow Vortex Induced Vibrations of Single Tapered Circular Cylinders: An Experimental Study. Applied Ocean Research, 42, 124-135. https://doi.org/10.1016/j.apor.2013.05.005

[17] Seyed-Aghazadeh, B., Carlson, D.W. and Modarres-Sadeghi, Y. (2015) The Influence of Taper Ratio on Vortex-Induced Vibration of Tapered Cylinders in the Crossflow Direction. Journal of Fluids and Structures, 53, 84-95. https://doi.org/10.1016/j.jfluidstructs.2014.07.014

[18] Sodano, H.A., Inman, D.J. and Park, G. (2004) A Review of Power Harvesting from Vibration Using Piezoelectric Materials. The Shock and Vibration Digest, 36, 197-205. https://doi.org/10.1177/0583102404043275 http://mesl.ucsd.edu/gupta/SHM/EHW/SVD\%202004-power\%20harvesting1.pdf

[19] Rostami, A.B. and Armandei, M. (2017) Renewable Energy Harvesting by Vortex-Induced Motions: Review and Benchmarking of Technologies. Renewable and Sustainable Energy Reviews, 70, 193-214. https://doi.org/10.1016/j.rser.2016.11.202

[20] Ueno, T. and Yamada, S. (2011) Performance of Energy Harvester Using Iron-Gallium Alloy in Free Vibration. IEEE Transactions on Magnetics, 47, 2407-2409. https://doi.org/10.1109/TMAG.2011.2158303

[21] Nakaguchi, H., Hashimoto, K. and Muto, S. (1968) An Experimental Study on Aerodynamic Drag of Rectangular Cylinders. Journal of Japan Society for Aeronautical and Space Sciences, 16, 1-5. (In Japanese) https://doi.org/10.2322/jjsass1953.16.1

[22] Okajima, A., Kimura, S., Katayama, T., Ohtsuyama, S. and Ojima, A. (1998) Fluid-Dynamic Characteristics of a Rectangular Cylinder with Various Width-to-Ratios in Wide Range of Reynolds Number. Journal of Structural Engineering, 44A, 971-977. (In Japanese) 


\section{Nomenclature}

$C_{n}=$ reduced mass-damping parameter of the system, $2 m \delta / \rho D H$

$D=$ depth of a prism in the flow direction

$f=$ vibration frequency of the prism in a flow

$f_{c}=$ characteristic frequency of the prism in a still water

$f_{\mathrm{n}}=$ natural frequency for vortex shedding from a fixed prism

$H=$ height of a prism normal to the flow direction

$L=$ span length of a prism

$I=$ length of a plate

$m=$ mass per unit length of the system

$N=$ number of data

$p_{\text {ave }}=$ average peak amplitude

$p_{\mathrm{i}}=$ peak amplitude

$p_{\text {rms }}=$ root-mean-square of the peak amplitude

$R e=$ Reynolds number, $U H / v$

$U=$ uniform flow velocity

$u=x$-component velocity

$V_{r}=$ reduced velocity, $U / f_{\mathrm{c}} H$

$V_{\text {rer }}=$ reduced resonance velocity, $U / f_{\mathrm{n}} H$

$V_{10.15}=$ reduced velocity at the $15 \%$ non-dimensional response amplitude of a prism

$y=$ displacement of the prism tip

$y_{\mathrm{rms}}=$ root mean square of displacement amplitude of the prism tip

$\delta=$ logarithmic decrement of the structural damping parameter of a prism

$\theta_{\mathrm{rms}}=$ root mean square of amplitude angle of the prism tip

$\eta_{\mathrm{rms}}=$ non-dimensional value of RMS response amplitude of cylinder vibrating in cross-flow direction, $\mathrm{y}_{\mathrm{rms}} / H(-)$

$v=$ kinematic viscosity of water

$\rho=$ water density 